\title{
Understanding women's experiences of distress during pregnancy in Dar es Salaam, Tanzania
}

\author{
S.F. KAAYA ${ }^{1 *}$, J.K. MBWAMBO ${ }^{2}$, M.C. SMITH FAWZI' ${ }^{3}$, H. VAN DEN BORNE ${ }^{4}$, H. SCHAALMA ${ }^{5}$ and \\ M.T. LESHABARI ${ }^{6}$ \\ ${ }^{1}$ Department of Psychiatry and Mental Health, Muhimbili University of Health and Allied Sciences, P.O. Box \\ 65023, Dar es Salaam, Tanzania \\ ${ }^{2}$ Department of Psychiatry and Mental Health, Muhimbili National Hospital, Dar es Salaam, Tanzania \\ ${ }^{3}$ Department of Global Health and Social Medicine, Harvard Medical School, Boston, USA \\ ${ }^{4}$ Department of Health Promotion, University of Maastricht, Maastricht, the Netherlands \\ ${ }^{5}$ Faculty of Psychology, University of Maastricht, Maastricht, the Netherlands \\ ${ }^{6}$ Department of Behavioural Sciences, Muhimbili University of Health and Allied Sciences, Dar es Salaam, \\ Tanzania
}

\begin{abstract}
Several studies show depression is common during pregnancy. However, there is limited information in Tanzania on the magnitude of perceived distress during pregnancy and meanings ascribed to such distress. A descriptive survey collected data using unstructured interviews from 12 traditional practitioners and 10 peri-urban women with previous pregnancy related mental health concerns identified using a depression vignette. The objectives were to describe the sources and characteristics of distress during pregnancy, and idioms of distress that could inform cultural adaptation of depression screening tools. Narrative analysis showed an emergent category of "problematic pregnancies" framed women's recollections of prolonged periods of sadness. This experience was qualified using various idioms of distress that were differentially emphasized depending on informant's perceived causes of health concern. The idiom kusononeka was consistently used to describe extreme sadness across causal categories and clustered with at least two typical features of major depression. This suggested existence of a construct with similarities to biomedical criteria for depression. "Thinking too much" emerged as a distinctive expression associated with prolonged sadness. Distinctive expressions of social functioning impairments were identified that can inform depression severity assessments. In conclusion, contextual inquiry into experiences of psychological distress showed distinct local idioms that clustered in patterns similar to symptoms of biomedical depressive episodes. Further studies to assess the utility of local idioms of distress and distress related functional impairment in depression assessment tools are warranted.
\end{abstract}

Key words: pregnancy, depression, distress idioms, explanatory models, Tanzania

\section{Introduction}

Evidence shows $10 \%-15 \%$ of women suffer depressive disorders during pregnancy or in the first postnatal year (Josefsson et al., 2001, Weissman \& Olfson, 1995). Studies indicate rates of depression during pregnancy are higher than or as high as in post-natal periods (Evans et al., 2001; Josefsson et al., 2001). Over a third of postnatal depressed women show high scores during pregnancy, suggesting persistence of pre-existing symptoms (Josefsson et al., 2001; Green \& Murray, 1994). While few, studies examining prenatal depressive morbidity in subSaharan Africa (SSA), show higher symptom endorsement (Fatoye et al., 2004) and severity (Cox, 1979) in pregnant compared to non-pregnant women in Nigeria and Uganda respectively. Adewuya et al. (2007) showed an $8.3 \%$ point prevalence of DSM-IV depressive

\footnotetext{
* Correspondence: Dr. Sylvia F.Kaaya; E-mail: skaaya@gmail.com
} 
disorder during late pregnancy in Nigerian antenatal clinic attendees. This rate was higher than the 1.3\% 12-month depression prevalence estimated in a Nigerian community-based sample of adults, where sex differentials in rates were minimal (Gureje et al., 2006). While several context specific sources of stress in SSA may challenge pregnant women's mental health, the HIV epidemic particularly increases the potential for distress in women screened for prevention of vertical transmission of HIV infections. Pregnant women in Zambia, informed for the first time of HIV positive status, showed higher percent endorsements on depressive symptoms than women with prior knowledge (Kwalombota, 2002). Studies show between 41\% (Rochat et al., 2006) and 43\% (Antelman et al., 2007) of HIV positive pregnant women endorse symptoms equivalent to major depression, in South Africa and Tanzania respectively.

While methodological and instrumentation differences may partly account for variations in observed rates of perinatal depressive morbidity, the influence of culture on experience and communication of emotional distress cannot be ruled out (Lutz, 1985; Beiser, 1985; Flaherty et al., 1998). There are limited qualitative studies from the culturally diverse communities in SSA of experiences with pregnancy related psychological distress. Depression prevalence studies in SSA, often rely on scrutiny of independent back translations for content and semantic equivalence of items when adapting standardized screening instruments (Cox, 1979; Fatoye et al., 2004; Adewuya et al., 2007). The inherent Euro-American ethnocentricity of depression screening tool items is however not addressed, raising questions regarding their cross-cultural utility (Kleinman, 1977; Lutz, 1985). Despite cross-cultural similarities in experiences of normal sadness, observed variations in the significance of depression as an illness category (Beiser, 1985) suggests the need for in-depth understandings of cultural influences on expression of depressive illnesses. Local models of illness in SSA resembling depressive morbidity such as kufungisisa in Zimbabwe (Patel, 1998) and guhahamuka in Rwanda (Bolton, 2001) are described by some studies while others, note an absence of conceptually equivalent depression terms (Swartz et al., 1985; Ihezue, 1989). Indigenous illness names tend to be explanatory categories, for example possession by spirits, rather than fixed illness categories, further confounding comparisons with biomedical syndromes. Finally, cross-cultural variations in ways of understanding the body and self result in different meanings ascribed to and expressions of psychopathology. For example, somatisation may be a distinctive feature of depressive experience in some cultures, while in others psychological expressions dominate (Kleinman, 1986). Cultural variations in what depression symptoms different groups emphasize may create item bias, with similarities in total scores on a screening tool, but variations occur in individual item scores across different cultural groups (van de Vijver \& Poortinga, 1997).

Formative research approaches are able to consider the unique conceptualizations and expressions of mood and hence can inform culture sensitive adaptation of mood measures (Halbreich et al., 2007). Such emic theoretical orientations in cross-cultural research pay attention to meanings particular groups ascribe to psychological phenomena (Lutz, 1985). The terms emic and etic support relativistic and universalistic positions respectfully; they originate from the field of linguistics and explore the origins of concepts under investigation in cross-cultural research. Relativistic positions study psychological phenomenon from the perspective of given cultural groups, arguing for uniqueness of experience that is often at odds with universalistic positions that consider emotions as biological phenomena with a limited number of common emotional experiences across cultural groups (Draguns et al., 2003). Both positions can be integrated when combining 
observations from qualitative understandings of illness experiences (an emic orientation) in the development of sensitive measuring instruments (predominantly an etic activity) (Draguns et al., 2003; Smit et al., 2006). These analyses report findings of a formative study that used emic theoretical approaches and aimed to understand experiences with psychological distress during pregnancy. The objective was to describe local idioms that would inform the cultural adaptation of a depression assessment tool.

\section{Materials and Methods}

\section{Study area}

The peri-urban ward of Chamazi with a relatively stable predominantly indigenous population of 8,868 residents, located $20 \mathrm{~km}$ from the Dar es Salaam City centre in Tanzania was purposefully selected. Residents of the Msufini and Mbande villages of the ward engaged in agricultural and fishing activities, and had minimal wealth diversity. Both villages had no electricity, and were each served by a primary school and clean water sources from protected public and private boreholes. A shared dispensary provided antenatal and other primary health care services. Health care providers had limited awareness of depressive disorder.

\section{Design and key informants selection}

Unstructured interviews collected data from traditional healers (TH) ("mganga wa jadi") and traditional birth attendants (TBA) ("mkunga wa jadi"), identified by village leaders as key providers of psychological health care. Six each of active TH and TBA were recruited as key informants. Traditional health practitioners identified, from a vignette illustrating core features of major depression (depressed mood for two weeks or more and loss of interest in usual activities), pregnant women attended in the past five years with health concerns similar to depression (previously affected women (PAW)) and sought their permission to be visited by the research team. Recruited PAW, though reticent on direct inquiry about psychological health problems, described personal experiences $(n=8)$ or providing care for an affected pregnant relative $(n=2)$, when presented with the depression vignette.

\section{Description of key informants}

Informants were of Bantu ethnicity, 69\% (11 of 16) from the predominant Zaramo linguistic sub-group. All TH were males of mean age (SD) 61.2 (31.4) years and TBAs females, of mean age (SD) 63.0 (16.9) years. All THs and two TBAs were married; two each of the remaining TBAs were widowed or divorced. Eight ( $n=5$ TBAs) traditional practitioners had never attended formal schooling. One TBA had been schooled for only four years and three THs had completed seven compulsory years of primary education. PAW informants were younger of mean age (SD) of 29.2 (7.9) years, four each were currently partnered or living alone (one widowed, one separated and two had never lived with a partner). Six and three PAW had complete and incomplete primary education respectively while one had never attended school. Informants lived in multifocal families with on average of 5.2 (range 1-9) household members.

\section{Data collection instruments and study procedures}


Ethical clearance was provided by the Muhimbili University of Health and Allied Sciences. Unstructured interview guides, explored types and perceived causes of pregnancy related health concerns and related expressions of distress. Interviews with PAW, probed recollected experiences of distress during pregnancy, idioms of distress and sources of support. Written and verbal informed consent was sought prior to interviews and audiorecords respectively. Two sociology graduates and the study investigators (JK, SK and ML) conducted one to two hour audio-recorded Kiswahili interviews in the homes of informants. Short field notes derived daily summaries and aided transcription of audio-records.

\section{Data analysis}

Transcribed narratives were entered as text and uploaded into the Qualitative Solutions and Research (1997) QSR NUD*1 ${ }^{\text {ST }}$ version 4.0 software to facilitate data management, open coding and retrieval of coded data. Axial coding was used to identify and describe clusters of idioms of distress.

\section{Results}

\section{Social-cultural Institutions and Problematic Pregnancies}

Initiation ceremonies were reported by informants to be the first formal social institution where women learnt about pregnancy and related norms and taboos that would ensure a safe delivery. Most PAW reported they had participated in initiation ceremonies $(n=9)$, which were often postponed when menarche occurred prior to completing primary education. Respected female mentors ("kungwi") provided instruction to initiates on women's roles as daughters and wives. These related mainly to social reproduction, family values in the kin group, norms and taboos related to sexual conduct and pregnancy. A public ceremony ("ngoma ya mkoleni"1), hosted by the initiates families gave visibility to graduates of rites of passage to womanhood and herald onset of relative seclusion in the family home. Women described assisting in household chores during periods of seclusion, lasting two to three years and ending at marriage. A second period of formal instruction was reported in the seventh month of a first pregnancy. Instruction included what to expect and how to behave during the birth process. At a public 'clothes giving' ceremony ("ngoma ya kupewa nguo"), pregnant women were formally introduced to the community of child bearing women. THs, usually male, played an active role in both the ceremonies including provision of protective amulets worn at all times henceforth by the woman.

Socio-economic, biomedical and supernatural factors emerged as causal categories for problematic pregnancies. Biomedical causes described as "God" given were distinguished from supernatural causes due to actions by persons or supernatural beings. Both were perceived less predictable of problematic pregnancy than socio-economic adversity. Characteristics of problematic pregnancy, protective or preventive measures and recollected idioms of distress are summarised in Table 1.

\section{Socio-economic adversity almost always results in "problematic pregnancies}

A 'coming-out' ceremony characterized by drums and dance (ngoma), that occurred under the Mkole tree that was associated with a woman's fertility. While timing of seclusion had changed due to the demands of formal education, narratives indicated its continued practice after completion of compulsory primary education (at age of about 14-15 years). 
Partners were expected to be the main source of support for pregnant women, with family assistance. Reluctance or inability to provide support was perceived to result in adversity, defined as lack of access to basic needs. Recollected distress due to poor support included bitterness in the heart/soul ("kujihisi uchungu moyoni/rohoni"), feeling sad as if in grief or mourning ("kusononeka"), or its converse, being unable to feel happy ("kuacha kucheka na kufurahi"). PAW also recalled having many thoughts ("kuwa na mawazo mengi"), lack of energy ("kukosa nguvu"), feeling tired most of the time ("kujisikia kuchoka muda mwingi"), and bodily pains ("maumivu ya mwili"). Inability to attend to personal grooming ("kutojali uonekano kimavazi na kujitunza") and social withdrawal or aloneness ("kujihisi mpweke" or "kunyongonyea", "kunyungurika") was also reported. Not eating well and weight loss were perceived due to lack of access to food, or bitter taste in the mouth ("uchungu mdomoni") rather than loss of appetite.

Table 1: Affected women's accounts of sources of pregnancy related problems, perceived causes and experiences

\begin{tabular}{|c|c|c|}
\hline Sources and protection & $\begin{array}{l}\text { Perceived } \\
\text { causes }\end{array}$ & $\begin{array}{l}\text { Experiences and idioms used to communicate } \\
\text { distress }\end{array}$ \\
\hline $\begin{array}{l}\text { Predictable "problematic } \\
\text { pregnancies": } \\
\text { No protection mentioned }\end{array}$ & $\begin{array}{l}\text { Socio- } \\
\text { economic } \\
\text { adversity }\end{array}$ & $\begin{array}{l}\text { Hunger; sadness as if grieved, feeling isolated and } \\
\text { alone, feeling bitterness in the heart/soul, having } \\
\text { many thoughts, loss of energy, feeling tired much of } \\
\text { the time, forcing oneself to do normal chores, lack of } \\
\text { attention to dressing, less laughter }\end{array}$ \\
\hline $\begin{array}{l}\text { Unpredictable } \\
\text { "problematic pregnancies" }\end{array}$ & Biomedical & $\begin{array}{l}\text { Palmer and gum pallor; fever; fatigue; rapid } \\
\text { heartbeats; shortness of breath; dizziness; swollen } \\
\text { feet; poor weight gain; fear; headache, having many }\end{array}$ \\
\hline $\begin{array}{l}\text { Protection: Adhering to } \\
\text { health care worker } \\
\text { recommendations; } \\
\text { Planning early for access to } \\
\text { health facility in case of } \\
\text { emergencies or help from } \\
\text { relative(s) }\end{array}$ & & thoughts. \\
\hline \multirow{2}{*}{$\begin{array}{l}\text { Protection: Adhering to } \\
\text { sanctioned pregnancy } \\
\text { related practices and } \\
\text { wearing protective } \\
\text { amulets }\end{array}$} & $\begin{array}{l}\text { Supernatural } \\
\text { Devil/spirit/sh } \\
\text { ade } \\
\text { possession }\end{array}$ & $\begin{array}{l}\text { Irritability, conflicts with partner, sadness as if } \\
\text { grieved, bad (or erotic) dreams, vaginal bleeding } \\
\text { prior to term, foetal death and/or still births, rapid } \\
\text { heartbeats, having many thoughts }\end{array}$ \\
\hline & $\begin{array}{l}\text { Active (being } \\
\text { bewitched) } \\
\text { and passive } \\
\text { effects of } \\
\text { malice, } \\
\text { jealousy } \\
\text { and/or envy } \\
\text { ("bad eye") }\end{array}$ & $\begin{array}{l}\text { Feeling physically unwell; headache; stomach ache, } \\
\text { fear, irritability and difficulties being with people; } \\
\text { having many thoughts; being overdue for delivery; } \\
\text { delayed delivery of the placenta; sadness as if } \\
\text { grieved; feeling like screaming or screaming without } \\
\text { being aware of doing so; feeling tired much of the } \\
\text { time, forcing oneself to do normal chores. }\end{array}$ \\
\hline $\begin{array}{l}\text { Protection: Avoid } \\
\text { breaching taboos }\end{array}$ & $\begin{array}{l}\text { Infringing } \\
\text { taboos related } \\
\text { to pregnancy }\end{array}$ & $\begin{array}{l}\text { Prolonged labour; retention of the placenta; } \\
\text { excessive bleeding during labour; still birth, } \\
\text { maternal death and fear of labour }\end{array}$ \\
\hline
\end{tabular}


Perceived biomedical causes of problematic pregnancy

Malaria, anaemia and poor diet were the main biomedical causes of problems during pregnancy mentioned. Malaria was attributed to mosquito bites transmitting an illness that caused fever ("homa2"), headaches, and sometimes abortions. Anaemia ("kukosa damu"), was experienced as a laboratory blood test result, palmer and gum pallor, fatigue ("kuchoka"), shortness of breath ("kukosa pumzi"), fear ("uwoga"), rapid heartbeats ("mapigo ya moyo kwenda kasi") and having many thoughts ("kuwa na mawazo mengi"). PAW defined nutritious foods as fish, meat, milk, green vegetables and fruits. Poor diet was reported to result in both anaemia and limited achievement of expected weight gains at antenatal assessments. While rarely acknowledged by informants, it is likely experiences such as increased heartbeat, shortness of breath and fatigue were related to having "many thoughts" about health or food security. The inability to predict problems due to biomedical causes is illustrated in the following quotation.

"...not having enough blood and insufficient body fluids both cause a lot of worry ....there is also malaria. Even without these problems, things can change very quickly close to delivery and a woman can lose a lot of blood and fluids and even die. These things cause many thoughts during pregnancy (PAW; 26 years old; married, housewife with four children; primary school graduate) Following dietary advice of health care providers and preparedness for the unexpected, were ways to prevent problematic pregnancies. Being prepared meant saving money for transport to a health facility, and having someone with, often an older female relative, who could help during late pregnancy and close to delivery.

\section{Pregnant women are vulnerable to supernatural influence}

Three reported sub-categories of supernatural causal factors included possession by devils ("shetani"3), spirits ("majini"), or shades ("mizimu"); bewitchment due to malice or the "evil eye" evoked by envy; and breaching pregnancy taboos.

\section{Spirit possession,' envy and malice cause "problematic pregnancies"}

Devils and spirits were described as invisible agents that were stepped on accidentally or "climbed on/into" to otherwise harm a person. Pregnant women were perceived to be particularly vulnerable to spirit possession, which was evidenced by conflict, altered mood and physical concerns. Symptoms included frequent conflicts with partners ("kutoelewana na mwenzi"), irritability ("kuudhiwa"), sadness as if grieved ("kusononeka"), bad or erotic dreams ("njonzi mbaya au za kuingiliwa"), rapid heartbeats ("mapigo ya moyo kwenda kwa kasi"), having many thoughts ("kuwa na mawazo mengi"), vaginal bleeding ("kutoka damu ukeni"), intrauterine foetal death ("mtoto kufia tumboni"), and still births ("kujifungua mtoto si riziki"). All informants reporting spirit possession $(n=3)$ described significant conflict with partners prior to pregnancy and spirit possession was always implicated as illustrated by the following quote:

\footnotetext{
${ }^{2}$ Literally translates to fever in English but the conceptual equivalence was broader ranging from chills with rigor to feeling unwell with generalized body malaise

${ }^{3}$ Shetani and jini were used interchangeably by PAW; TH perceived jini to be a category of spirits, with intentions not always considered evil as often implied by the term shetani. In this largely Muslim community, users of the term may have implied the more negative connotation of shetan as used in Koranic vocabulary where the term can more closely be translated to mean the Devil.
} 
"... This state of feeling sad as if grieved (kusononeka) is due to being irritated when there is no cause. It is the result of a bad bug that is "jini mahaba". It makes a man or woman sulk for no reason and the two can split up... because the jini wants an exclusive relationship with the woman, and can also ruin the pregnancy." (PAW, 36 years old, separated with four children; no formal education)

Envy from others during pregnancy was a recurring theme. Active acts of jealousy with malicious use of witchcraft, though distinguished from more passive influences of envious thoughts, were described to have similar effects. These included low energy ("mwili kukosa nguvu"), headache or stomach-ache ("maumivu ya kichwa au tumbo"), fear ("uwoga"), irritability ("kuudhiwa"), being unable to interact with people ("kutoweza kujumuika na watu"), many thoughts ("mawazo mengi"), post delivery date pregnancies ("kufungwa mimba"), feeling sad and as if grieved ("kusononeka"), wanting to scream, or screaming without being aware of doing so ("kuhisi kupiga kelele" or "kupiga kelele bila kujijua"), and needing to force oneself to do household chores ("kujilazimisha kufanya shughuli za kawaida"). The following quotation illustrates attribution of ill health to envious thoughts in a situation a PAW could not communicate concerns to her partner:

"...I went to the hospital because of the headaches; ...they took some blood and urine tests and everything was normal. I got along well with my co-wife, but I worried because she has no children, maybe her envy was the cause? ... I talked to my father... we agreed he suggests to my husband that my illness may be due to "mizimu" (shades), and that I should move to my parent's home for care.." (PAW; 26 years old; second wife with four children; primary school graduate)

Amulets worn during pregnancy were perceived to prevent spirit possession and bewitchment. Interventions in the event of spirit possession included healing rituals mediated by THs who made known the demands of spirits to partners and family members. Responses would include offerings of gifts (often clothes and jewellery) to the affected woman to appease spirits. Women were also instructed to take good care of "identifying items" such as clothes, hair, the after birth, and dried remnants of the umbilical cord that could be used to harm, if acquired by persons with malicious intentions.

\section{Breaching pregnancy related taboos causes problems during delivery}

Most described taboos protected against poor pregnancy outcomes. The infidelity taboo for example, was most consistently described and applied to both partners during pregnancy. Described consequences of infidelity included prolonged labour, still births, excessive bleeding during child birth, retention of the placenta, and maternal death. "Mixing bodily fluids" of different persons was perceived harmful to the foetus or at childbirth as illustrated by the following quote from a TBA:

"....once you make a person pregnant, stick to that person until a healthy baby is born--- our children don't listen these days and continue other relationships and as a result, women get problems during delivery. The Zaramo say every person with his own fluid and it is not good to mix fluids". (TBA; 75 years old, divorced with four children; no formal education)

PAW described limitations in protecting their unborn infants and themselves from consequences of infidelity, due to limitations in their control over sexual behaviours of partners. They also described fear of impending childbirth should one unwittingly breach taboos $(n=3)$ and distress when accused by TBAs of breaching taboos when complications of labour occurred $(n=2)$. 


\section{"Problematic pregnancies" and clustering of expressions of distress}

Prolonged sadness was not perceived to occur for no reason, and was often attributed to malaise, pain (abdominal and/or headaches), or socio-economic adversity. Idioms used to qualify experiences of unhappiness included sadness as if grieved and bitterness in the heart or soul. Sadness as if grieved ("kusononeka") was consistently described as an experience more extreme than normal sadness, prohibiting abilities to perform household chores by making the feet heavy and numb and slowing down movements. Interpersonal difficulties (being unable to interact with people and conflicts with intimate partners) were not surprisingly reported with irritability, tenseness, and headache and whirling or heat sensations in the head, as illustrated in the following PAW's narration:

“.... I don't know how I felt. My head was not right, I had pain and tension, and I was not feeling good and was angry when I was with people. Sometimes I was so irritated by people and felt like screaming..." (PAW; 26 year old; second wife with four children; primary school graduate)

Experiences of low energy and being frequently tired were the only idioms of distress that reportedly could occur for no reason. Accounts of feeling tired all the time consistently cooccurred with having many thoughts and varying impairments in social functioning. The following quotation is illustrative:

".... Feeling tired all the time and alone causes many thoughts; the life inside a woman's home is sometimes very difficult. Women think about whether they have eaten well (recommended nutritious foods), have enough food, have clothes for themselves and the baby, and these worries bring more weakness, sadness and there is pain in the whole body" (PAW4, 40 year old widow, primary school graduate with 7 children)

As illustrated in the quotation above, having many thoughts was frequently described in response to pregnancy related worries and concerns, regardless of perceived causes, and as a reason for pain and feeling frequently tired.

\section{Discussion}

Women and traditional practitioner key informants all perceived pregnancy as a normal phenomenon and positive outcomes (healthy infant and mother) were generally anticipated. Good (1994) suggests that just as biomedical diagnostics and treatment procedures construct a particular number of objects for transformation through therapeutic interventions, nonmedical healing practices similarly construct objects for transformation through both protective and healing interventions. Traditional initiation and pregnancy related institutions, provided norms and normative values for practices facilitating a "safe" pregnancy, hence objectifying agents of future problems during pregnancy. An emergent category, the "problematic pregnancy" ("ujauzito wa matatizo), framed women's recollected experiences of distress. PAW, when prompted, were willing to acknowledge, express and qualify experiences of sadness during pregnancy. A consistent idiom expressing extreme sadness, "kusononeka", sometimes associated with cognitive and behavioural idioms of distress and always with impairments in social functioning emerged. Finally reported idioms of distress clustered in certain patterns, within and across perceived causes of problematic pregnancy.

In these analyses, women often attributed depressed mood to physical complaints or socio-economic difficulties. Similar attributions of depressed mood were shown in a study that recruited informants from two regions of Tanzania who represented 35 tribal affiliations 
(Whyte, 1991). The emphasis given to somatic and cognitive complaints when describing emotional distress within emergent causal categories of "problematic pregnancies" may suggest absence of a mind-body division, implied to be a Western Cartesian dichotomy (Scheper-Hughes \& Lock 1987; Wen 1998). However, PAW's initial reticence to discuss "psychological health" problems, may suggest informants did make mind-body illness distinctions. Kohrt \& Harper (2008) explored the ethnopsychology of mind-body dichotomies in Nepal and showed, rather than a lack of mind-body division, emphasis on bodily presentations of psychological distress reflects presence of such divisions and the stigma associated with mental illness. These analyses did not allow exploration of influences of stigma on expressions of distress, however, negative attitudes towards mental disorders in Tanzanian communities have been reported (Whyte, 1991), as well as high felt and enacted stigma scores in facility-based ambulatory patients with mental disorder (Philip, 2003).

Reported idioms of distress clustered in distinctive patterns within perceived causes of problematic pregnancy suggesting influences of causal explanatory models on idioms of distress emphasized in women's recollections. For example, accounts of sadness occurred with feelings of bitterness and loneliness when socio-economic factors were perceived to cause health problems. There is evidence that shows stressful life events are common and closely associated with depression in low income urban women in SSA (Broadhead et al., 2001). With spirit possession attribution however, sadness clustered with irritability and interpersonal conflict, particularly with partners. Studies show conflicts with intimate partners can both be a result of perinatal depression (Kazi et al., 2006; Hart et al., 2006), and a risk factor for perinatal depression across diverse cultural settings (Farber et al., 1996; Bolton et al., 1998; Pajulo et al., 2001). In the context of low socio-economic status and multifocal family structures, the need to foster supportive networks is perhaps greater. Attribution of conflict to possession states may be a distinctive expression of distress associated with interpersonal conflicts; particularly when there is some evidence that questioning by women of aspects of intimate relationships such as an infidelity may not be acceptable (Lary et al., 2004). This interpretation is supported by the symbolism in TH mediated treatment of spirit possession; where an acceptable and safe environment is provided for expression of discontent through the medium of spirits. Features of fear and anxiety predominated accounts of idioms of distress when biomedical causes of pregnancy related problems were reported. Such responses were perhaps realistic, given the high rate of maternal mortality in Tanzania estimated at 43.1-123 per 100,000 women aged 15-49 years (Mswia et al., 2003), in the community and 754 maternal deaths /100,000 live births (Alloo, 1994) at a tertiary referral health facility serving the study area.

Symptoms clustering with sadness across more than one perceived cause included low energy or excessive fatigue and loss of interest in activities, were similar to ICD-10 (WHO, 1992) typical features of depressive episodes. Having many thoughts a symptom that clustered with sadness across all the causal categories is not an ICD-10 typical or other feature of depression and should be considered a culturally distinctive expression of depression. Thinking too much was also shown to be a significant idiom of distress in depressed Zimbabwean women in (Patel, 1998). Some described somatic concerns not considered ICD-10 features of major depression, are shown to be common presentations of depression in primary care settings (den Boer et al., 2002; Escobar et al., 2006) and Kelly et al. (2001) reported amplification of physical health symptoms in depressed pregnant women. Subjective accounts of difficulties in social role functioning (household chores, and social 
interaction) and personal grooming, provided context specific information on functional impairments to guide assessment of depression severity (WHO, 1992).

There are limitations in generalizing accounts of informants from a single locality given the 125 linguistic groups of Tanzania. It can, however, be argued that the Bantu are the largest ethnic group in the country, with similarities in customs across linguistic sub-groups. Common use of Kiswahili throughout Tanzania may also facilitate diffusion of cultural structuring of pregnancy experiences and related meanings and expressions of distress. The five year recall timeframe required of PAW informants was a second limitation. It was anticipated that experiences of significant distress would be recalled, the potential for recall bias cannot be ruled out. The process of selection of PAW informants most able to provide the required information and triangulation by comparing and contrasting accounts from different types of informants may also increase the validity of study findings. Despite these limitations, information is provided that can inform adaptation of tools for future quantitative study of depression.

In conclusion, contextual inquiry into experiences of psychological distress showed distinct local idioms that clustered in patterns similar to symptoms of biomedical depressive episodes. Further studies to assess their utility in depression assessment tools are warranted.

\section{Acknowledgements}

Authors thank study participants and research assistants, particularly Ms. Violet Mrema. Institutional support from the Muhimbili University and funding support from the Canadian International Development and Research Centre made these analyses possible.

Received 3 December 2009

Revised 10 December 2009

Accepted 11 December 2009

\section{References}

Adewuya, A.O., Ola, B.A., Aloba, O.O., Dada, A.O. \& Fasoto, O.O. (2007) Prevalence and correlates of depression in late pregnancy among Nigerian women. Depression and Anxiety 24, 15-21.

Alloo, F., (1994) Maternal mortality and morbidity. Women's reproductive health in Tanzania. Women's Global Network for Reproductive Rights 46, 12.

Antelman, G., Kaaya, S., Wei, R., Mbwambo, J., Msamanga, G.I., Fawzi, W.W. \& Fawzi, M.C. (2007) Depressive symptoms increase risk of HIV disease progression and mortality among women in Tanzania. Journal of Acquired Immune Deficiency Syndrome 44, 470477.

Beiser, M. (1985) A study of depression among traditional Africans, urban North Americans, and South Asian refugees. In Ed. Kleinman A, Good B. Culture and Depression. Berkeley: University of California Press, p. 272.

den Boer, J.A., Evans, D.L., Lee, S. \& Salin-Pascual, R. (2002) Unravelling the diagnostic clues of depression and GAD: The primary care challenge. Psychopharmacology Bulletin 36, (Suppl. 2), 150-157.

Bolton, P. (2001) Local perceptions of the mental health effects of the Rwandan genocide. Journal of Nervous and Mental Disorders 189, 243-248. 
Broadhead, J., Abas, M., Sakutukwa, G. K., Chigwanda, M. \& Garura, E. (2001) Social support and life events as risk factors for depression amongst women in an urban setting in Zimbabwe. Social Psychiatry and Psychiatric Epidemiology 363,115-122.

Cox, J.L. (1979) Psychiatric morbidity and pregnancy: a controlled study of 263 semi-rural Ugandan women. British Journal of Psychiatry 134, 401-405.

Draguns, J.G., \& Tanaka-Matsumi, J. (2003) Assessment of psychopathology across and within cultures: issues and findings. Behaviour Research and Therapy 41, 755-776.

Escobar, J. I., Interian, A., Diaz-Martinez, A. \& Gara, M. (2006) Idiopathic physical symptoms: a common manifestation of psychiatric disorders in primary care. CNS Spectrums, 11, 201-210.

Evans, J., Heron, J., Francomb, H., Oke, S. \& Golding J. (2001) Cohort study of depressed mood during pregnancy and after childbirth. British Medical Journal 323, 257-260.

Fatoye, F.O., Adeyemi, A.B. \& Oladimeji, B.Y. (2004) Emotional distress and its correlates among Nigerian women in late pregnancy. Journal of Obstetrics and Gynaecology, 245, 504-509.

Green, J. \& Murray, D. (1994) The use of the Edinburgh Postnatal Depression Scale in research to explore the relationship between antenatal and postnatal dysphoria. In Cox J, Holden J eds. Perinatal Psychiatry. London: Gaskell, p. 180.

Gureje, O., Lasebikan, V.0., Kola, L. \& Makanjuola, V.A. (2006) Lifetime and 12-month prevalence of mental disorders in the Nigerian Survey of Mental Health and Wellbeing. British Journal of Psychiatry 1885, 465-471

Halbreich, U., Alarcon, R.D., Calil, H., Douki, S., Gaszner, P., Jadresic, E., Jasovic-Gasic, M., Kadri, N., Kerr-Correa, F., Patel, V., Sarache, X. \& Trivedi, J.K. (2007) Culturallysensitive complaints of depressions and anxieties in women. Journal of Affective Disorders, 102, 159-176.

Hart, R. \& McMahon, C.A. (2006) Mood state and psychological adjustment to pregnancy. Archives of Women's Mental Health 9, 329-337.

Ihezue, U.H. (1989) The influence of socio-cultural factors on symptoms of depression. In Ed. Peltzer K, Ebigbo P. Clinical Psychology in Africa. Chuka Printing: Enugu, Nigeria p. 217.

Josefsson, A., Berg G., Nordin C. \& Sydsjö, G. (2001) Prevalence of depressive symptoms in late pregnancy and post-partum. Acta Obstetricia et Gynaecologica Scandinavica 80, 251255.

Kazi, A., Fatmi, Z., Hatcher, J., Kadir, M.M., Niaz, U. \& Wasserman G.A. (2006) Social environments and depression among pregnant women in urban areas of Pakistan: Importance of social relations. Social Science and Medicine 63, 1466-1476.

Kelly, R. H., Russo, J. \& Katon, W. (2001) Somatic complaints among pregnant women cared for in obstetrics: normal pregnancy or depressive and anxiety symptom amplification revisited? General Hospital Psychiatry 23, 107-113.

Kleinman, A.M. (1986) Social Origins of Distress and Disease: Depression, Neurasthenia and Pain in Modern China. Yale University Press, New Haven CT.

Kleinman, A. (1977) Depression, somatisation, and the "new cross-cultural psychiatry." Social Science and Medicine 11, 3-9.

Kohrt, B.A. \& Harper, I. (2008) Navigating diagnoses: Understanding mind-body relations, mental health, and stigma in Nepal. Culture Medicine and Psychiatry 324, 462-491.

Kwalombota, M. (2002) The effect of pregnancy in HIV-infected women. AIDS Care 14, 431433. 
Lary, H., Maman, S., Katebalila, M., McCauley, A. \& Mbwambo, J. (2004) Exploring the association between HIV and violence: young people's experiences with infidelity, violence and forced sex in Dar es Salaam, Tanzania. International Family Planning Perspectives 304, 200-206.

Lutz C. (1985) Depression and the translation of emotional worlds. In Ed. Kleinman A, Good B. Culture and Depression. University of California Press: Berkeley

Mswia, R., Lewanga, M., Moshiro, C., Whiting, D., Wolfson, L., Hemed, Y., Alberti, K.G., Kitange, H., Mtasiwa, D. \& Setel, P., (2003) Community-based monitoring of safe motherhood in the United Republic of Tanzania. Bulletin of the World Health Organization 81, 87-94.

Patel, V. (1998) Culture and Common Mental Disorders in Sub-Saharan Africa. Maudsley Monographs 41, Psychology Press, East Sussex.

Phillip, F. (2003) Stigma towards mental illness: The experience of mentally-ill patients and relatives attending the psychiatric unit, Muhimbili National Hospital. MMed Dissertation, University of Dar es Salaam, Tanzania

Rochat, T.J., Richter, L.M., Doll, H.A. \& Buthelezi, NP. (2006) Depression amongst pregnant rural South African women undergoing HIV Testing. Journal of the American Medical Association 29512, 1376-1378.

Scheper-Hughes, N. \& Lock M.M. (1987) The mindful body: A prolegomenon to future work in medical anthropology. Medical Anthropology Quarterly 11, 6-41.

Smit, J., van den Berg, C.E., Bekker, L.G., Seedat, S. \& Stein, D.J. (2006) Translation and crosscultural adaptation of a mental health battery in an African setting. African Health Sciences 6, 215-222.

Swartz, L., Ben-Arie, O. \& Teggin, A.F. (1985) Sub cultural delusions and hallucinations. Comments on the Present State Examination in a multi-cultural context. British Journal of Psychiatry 146, 391-394.

van de Vijver, F.J.R. \& Poortinga, Y.H. (1997) Towards and integrated analysis of Bias in cross-cultural assessment. European Journal of Psychological Assessment 13, 29-37.

Wen, J.K. (1998) Folk belief, illness behaviour and mental health in Taiwan. Chang Kengi Hsueh Tsa Chih 211, 1-12.

Weissman, M.M. \& Olfson, M. (1995) Depression in women: Implications for health care research. Science 269, 799-801

Whyte, S.R. (1991) Attitudes towards mental health problems in Tanzania Acta Psychiatrica Scandinavica Suppl. 364, 59-76.

WHO (1992) The ICD-10 Classification of Mental and Behavioural Disorders: Clinical descriptions and diagnostic guidelines. Oxford University Press: Madras 